\title{
SELECTION EQUILIBRIA IN A MULTIALLELE SINGLE-LOCUS SETTING
}

\author{
P. J. HUGHES and E. SENETA* \\ Australian National University, Canberra
}

Received 2.i.75

\begin{abstract}
SUMMARY
The aim of the paper is to clarify and unify various well-known results in the setting mentioned in the title, since some of these results are either inaccurate, or incomplete; or refer to different concepts bearing the same name. Emphasis is given to the general case, i.e. we include the situation of singular fitness matrix, a topic which is generally avoided. We proceed by using a specific generalized inverse of the fitness matrix. A full bibliography is given.
\end{abstract}

\section{InTRODUGTION}

WE deal with a diploid single locus situation, at which locus any one of $k$ alleles $A_{i}, i=1, \ldots, k$ can occur. The fitness of individuals of genotype $A_{i} A_{j}$, assumed to depend only on this genotype structure, is denoted by $w_{i j}$. Mating is assumed random. We shall denote by $W=\left\{w_{i j}\right\}, i, j=1, \ldots, k$ the matrix (symmetric and with non-negative entries) whose elements are the fitnesses; and by $p_{i}, i=1, \ldots, k$ the allele frequencies at the stage of zygote formation of the present generation. Primes will be used to denote quantities at the corresponding stage in the following generation. It is well known that the allele frequencies in the following generation are related to those of the present generation by the transformation

where

$$
p_{i}^{\prime}=\bar{w}_{i} p_{i} / \mu, \quad i=1, \ldots, k,
$$

$$
\bar{w}_{i}=\sum_{j=1}^{k} w_{i j} p_{j}, \quad \mu=\sum_{i, j} p_{i} w_{i j} p_{j}=\sum_{i} \bar{w}_{i} p_{i} .
$$

Thus $\mu$ denotes the mean fitness of individuals (in the current generation).

A frequency vector $\boldsymbol{p}=\left\{p_{i}\right\}, i=1,2, \ldots, k$, where $p_{i} \geqq 0$ for all $i$, and

$$
\sum_{i=1}^{k} p_{i}=p^{T} 1
$$

is said to represent a state of equilibrium of the genetic system if $\boldsymbol{p}^{\prime}=\boldsymbol{p}$. From (1) it follows that necessary and sufficient for this, providing $\boldsymbol{p}>0$, is

i.e.

$$
\mu=\bar{w}_{i}\left(=\sum_{j=1}^{k} w_{i j} p_{i}\right), \quad i=1, \ldots, k
$$

$$
\mu \mathbf{1}=W p
$$

where $\mu=\mu(\boldsymbol{p})$ is the mean fitness at $p$. An equilibrium vector or " point" $p$ is said to be internal, or non-trivial, if apart from $p \geqq 0, p^{T} \mathbf{1}=\mathbf{1}$ and (1),

* All requests and correspondence to this author, at Department of Statistics (School of General Studies). 
it satisfies $p_{i}>0$ all $i$. Clearly an internal equilibrium point corresponds to a $p>0$ satisfying (2) for some $\mu$. An equilibrium vector is said to represent a boundary equilibrium if it contains some zero entries, i.e. is an equilibrium vector but not an internal one; it is then said to be a trivial equilibrium point.

Most interest centres on internal equilibrium points, about which questions concerning conditions for their $(a)$ existence, $(b)$ uniqueness and (c) stability are asked. (We shall later deal briefly with these questions also in relation to boundary equilibria, the situation being formally analogous.) A number of papers dealing with these topics has appeared.

As regards the question of existence, while it seems to be implicitly recognized that more than one internal equilibrium point can exist (Mulholland and Smith, 1959), there exist definite authoritative statements which suggest the contrary. Specifically, Mandel (1959, p. 290) states that in order that an internal equilibrium exist, it is necessary that all $W_{i}$, $i=1, \ldots, k$ (where

$$
\left.W_{i}=\sum_{j} W_{i j}, W_{i j} \text { being the }(i, j) \text { cofactor of } W\right)
$$

have the same sign; and that there can be at most one such non-trivial state of equilibrium. This last is perpetuated, e.g. in the book of Ewens (1969, p. 11). In a recent paper Mandel (1970, p. 842) asserts that it was in fact demonstrated in his earlier note that the condition: that all $W_{i}$ be non-zero, and all have the same sign, is necessary and sufficient for existence of internal equilibrium. While these statements are true if the matrix $W$ is assumed non-singular (as Weir (1970) is one of few to note explicitly), they are decidedly false if $W$ is singular, as we shall demonstrate. In fact, very little attention as a whole seems to have been paid specifically to the case (as a separate situation) where the fitness matrix is singular, which possibility is at least mentioned by Tallis (1966); and one of our aims is to rectify this. This is particularly important in relation to the problem of uniqueness of internal equilibrium points.

The problem of stability of an internal equilibrium point, if it exists, has been rather more successfully studied, the basic references here being Kingman $(1961 b)$, on the one hand, who gives a necessary and sufficient criterion in terms of the eigenvalues of $W$; and on the other hand necessary and sufficient conditions of various kinds, but all of determinantal form, are summarised and demonstrated to be equivalent in Mandel's (1970) note (in the last connection, see also the book of Crow and Kimura, 1970, pp. 275-277). Confusion here has arisen because (apart from the differences in approach already mentioned) in each of these two schools the definition of "stable point" is, in fact different. Kingman uses a definition of stability rather more general than the other authors. As a result, the necessary and sufficient conditions for stability obtained by each school do not refer to the same notion, and hence are not equivalent, as is suggested by some texts.

There is one result, however, in the situation of current interest, about which there is no dispute, and to which we shall refer, viz. that mean fitness is non-decreasing from generation to generation, i.e. if the probability vectors $p^{\prime}$ and $p$ are related by (1), then

$$
\mu\left(\boldsymbol{p}^{\prime}\right) \geqq \mu(\boldsymbol{p}) .
$$


This result has been obtained by several authors, viz. Scheuer and Mandel (1959), Mulholland and Smith (1959), Atkinson, Watterson and Moran (1960) and Kingman (1961a). In fact, $\mu\left(\boldsymbol{p}^{\prime}\right)>\mu(\boldsymbol{p})$ unless $\boldsymbol{p}$ is an equilibrium point.

The aim of this paper is to attempt to resolve and unify some of the ambiguities mentioned above, with emphasis on the situation when $W$ is singular. In the interests of brevity and readability, we give some mathematical consideration only at those points, where the mathematics is not included in the references, or the Mathematical Appendix.

A full bibliography for the subject-matter is given for the reader's convenience.

\section{Existence AND UNiQueness of INTERNAL EQUilibria}

We shall make use here of the identity that for any square matrix $A$, whether singular or not

$$
A(\operatorname{adj} A)=(\operatorname{adj} A) A=(\operatorname{det} A) I,
$$

where adj and det are, respectively, contractions for adjoint and determinant. If we put $A=W,(2)$ gives

$$
\mu(\operatorname{adj} W) \mathbf{1}=(\operatorname{adj} W) W p=(\operatorname{det} W) p,
$$

where $(\operatorname{adj} W) \mathbf{1}=\left\{W_{i}\right\}$. Thus if $(\operatorname{det} W) \neq 0$, the statements of Mandel mentioned above are certainly true.

However, if $\operatorname{det} W=0$, clearly* each $W_{i}=0$. However, internal equilibria are still possible. For example if

$$
W=\left[\begin{array}{lll}
1 & 3 & 2 \\
3 & 1 & 2 \\
2 & 2 & 2
\end{array}\right]
$$

then the positive probability vector $(t, t, 1-2 t)$, where $t$ is in the interval $0<t<\frac{1}{2}$, satisfies (2) for each such $t$, with $\mu=2$; and hence defines a whole continuum of internal equilibrium points.

It will be shown in the next section, however, that there can be at most one internal equilibrium point which is stable in a strict sense; and it is only internal equilibrium points of this kind which seem to be of interest in population genetics.

However, in general, it is still possible in theory to find all equilibrium points, whether internal or boundary, stable or unstable, finite or infinite in number, by making use of some results concerning generalised inverses in the sense of Rao (1965, pp. 24-26) in connection with attempting solution of (2) for $\boldsymbol{p}$. Suppose that the rank of $W$ is $r, r \leqq k$. Then merely by changing the labelling on various alleles and writing $W$ accordingly, we can write $W$ in the form

$$
W=\left[\begin{array}{ll}
A_{11} & A_{12} \\
A_{21} & A_{22}
\end{array}\right],
$$

* Except in the uninteresting case where $\mu=0$. 
where $A_{11}$ is $r \times r$, symmetric and of full rank, and hence non-singular (see Appendix). The matrix

$$
W^{-}=\left[\begin{array}{ll}
A_{11}^{-1} & 0 \\
0 & 0
\end{array}\right]
$$

is a generalised inverse of $W$, and hence every solution $p$ of (2), whether a probability vector or not, is given by

$$
\boldsymbol{p} / \mu=W^{-} \mathbf{1}+(I-H) z,
$$

where $z$ is an arbitrary vector and $H=W^{-} W$;

$$
=\left[\begin{array}{c}
A_{11}^{-1} 1 \\
0
\end{array}\right]+\left[\begin{array}{c}
-A_{11}^{-1} A_{12} z_{2} \\
z_{2}
\end{array}\right]=\left[\begin{array}{c}
A_{11}^{-1}\left(1-A_{12} z_{2}\right) \\
z_{2}
\end{array}\right]
$$

where $z_{2}$ is arbitrary and $z$ has been appropriately partitioned into two components $z_{1}$ and $z_{2}$, where $z_{2}$ is $(k-r) \times 1$, and arbitrary (if not of zero length, when $k=r$ ).

If we consider only equilibria for which $\mu \neq 0$, it follows that an equilibrium point $\boldsymbol{p}$ (if any exist) must be of the form

where

$$
p=\frac{1}{g\left(z_{2}\right)}\left[\begin{array}{c}
A_{11}^{-1}\left(1-A_{12} z_{2}\right) \\
z_{2}
\end{array}\right],
$$

$$
g\left(z_{2}\right)=1^{T} A_{11}^{-1}\left(1-A_{12} z_{2}\right)+1^{T} z_{2},
$$

and at such an equilibrium value

$$
\mu(p)=1 / g\left(z_{2}\right) .
$$

Conversely, if in the form (4), a $z_{2}$ exists such that

$$
z_{2} \geqq 0, A_{11}^{-1}\left(1-A_{12} z_{2}\right) \geqq 0 \text {, and } g\left(z_{2}\right)>0,
$$

then the $\boldsymbol{p}$ related to $z$ by (4) defines an equilibrium state, which may be internal or boundary.

For an internal equilibrium state one needs a $z_{2}$ satisfying

$$
z_{2}>0, A_{11}^{-1}\left(1-A_{12} z_{2}\right)>0 \text {. }
$$

Suppose such a state and so such a $z_{2}$ exists. Then if we put $z_{2}^{*}=z_{2}+\delta$ where $\delta$ is a vector with sufficiently small entries and assume that $r<k$ (so that we are in fact dealing with a genuine singular $W$ ) it will follow that $z_{2}^{*}$ also satisfies

$$
z_{2}^{*}>0, A_{11}^{-1}\left(1-A_{12} z_{2}^{*}\right)>0,
$$

so $z_{2}^{*}$ defines an internal equilibrium also. Moreover

$$
g\left(z_{2}\right)=g\left(z_{2}^{*}\right)=\mathbf{1}^{T} A_{11}^{-1} \mathbf{1} .
$$

[See Appendix.]

Hence, in the case of singular $W$, there is either no internal equilibrium point; or there are uncountably many, with the same fitness value.

In the latter case, regarding the $z_{2}$ as points in $k-r$ dimensional Euclidean space, it follows that those $z_{2}$ defining internal equilibrium vectors $p$ form an open set; and that there is a I: I correspondence between such $z_{2}$ and $p$. 
As an example of the former case consider the (nominally) 3-allele situation, where

$$
W=\left[\begin{array}{lll}
\beta & \alpha & 0 \\
\alpha & \gamma & 0 \\
0 & 0 & 0
\end{array}\right], \quad \alpha, \beta, \gamma>0 .
$$

It is seen that for this case, the three simultaneous equations given by (2) are inconsistent, in view of the requirement that $\mu>0$.

\section{STABility AND UNIQUeNESS OF INTERNAL EQUiLIBRIA}

(i) Internal stationary points of fitness function

It is well known that if we use the method of Lagrange multipliers to seek stationary points of the quadratic form $p^{T} W p$ under the linear constraint $p^{T} 1=1$, we obtain the equations (2), whether or not $W$ is singular. Let us assume that an "internal" solution $\hat{p}>\mathbf{0}$ exists (there will be at most one if $W$ is non-singular); by Section 1 it will also be an equilibrium point. Since we know that, from generation to generation, the mean fitness $p^{T} W p$ is strictly increasing unless $\boldsymbol{p}$ is an equilibrium point, such a stationary point will be stable if it corresponds a maximum, and unstable otherwise. To investigate the issue, we consider a perturbation to the vector $\hat{\boldsymbol{p}}: \hat{\boldsymbol{p}}+\boldsymbol{\delta}$, such that $\delta \neq 0, \delta^{T} \mathbf{1}=0$, and well-known arguments which need not be repeated here yield finally that $\hat{p}$ is a strict maximum if and only if

$$
\delta^{T} W \delta<0 \text { for each } \delta \neq 0, \delta^{T} \mathbf{1}=0 .
$$

In view of the sequel, it is appropriate to speak of the more general possibility, of $\hat{\boldsymbol{p}}$ being a weak maximum, which occurs if and only if

$$
\delta^{T} W \delta \leqq 0 \text { for each } \delta \neq 0, \delta^{T} 1=0 .
$$

In this case it is possible that a small non-zero perturbation $\delta$ of $\hat{\boldsymbol{p}}$ will result in movement to a distinct point $p^{*}=\delta^{*}+\hat{p}$, at which the fitness value is the same as at $\hat{p}$; hence $p^{*}$ will also be a point of maximal fitness, and since any other perturbation of the kind $\delta=c \delta^{*}$, where $c$ is a positive constant, will produce the same effect, $\hat{p}^{*}$ will in fact be an accumulation point of internal vectors $p$ at each of which the fitness is also maximised, and so each of which is also an equilibrium point. This accords completely with the reasoning of Section 2, and clearly cannot happen if $W$ is nonsingular. Thus if $W$ is non-singular, if $\hat{\boldsymbol{p}}$ is a weak maximum, it is also a strict maximum.

\section{(ii) Stability}

We call an internal equilibrium ( $=$ stationary) point $\hat{p}$ stable (a better term would be strictly stable), if and only if (6) obtains. Note that at most one such point can exist since it is a point of strict maximum of $p^{T} W_{p}$. Also, were $W$ assumed singular, Section 2 reveals that $\hat{p}$ would be an accumulation point of other internal equilibrium points, at which the mean fitness achieves equilibrium-stationary values of the same magnitude. Hence strict stability may only occur when $W$ is non-singular, and is therefore not the appropriate concept to be used for arbitrary $W$. 


\section{(iii) Semi-stability}

We call an internal equilibrium ( = stationary) point $\hat{\boldsymbol{p}}$ semi-stable (a better term would be: weakly stable), if and only if (7) obtains. This condition renders $\hat{p}$ a point of maximum of $\boldsymbol{p}^{\prime} W \boldsymbol{p}$ over all $\boldsymbol{p}>\mathbf{0}, \boldsymbol{p}^{T} \mathbf{1}=1$, but there may be other such points. Necessary and sufficient conditions for internal semi-stability have been examined by Kingman (1961 $b$ ) -although he also, unfortunately talks of "stability". Recall that $W$ is (a) a symmetric matrix, having therefore only real eigenvalues; and $(b)$ a non-negative matrix, which by Perron-Frobenius theory has a (not necessarily unique) dominant root which can be taken as non-negative, and, indeed, positive (unless $W=0$, a trivial case) in view of symmetry (Seneta, 1973). Kingman's necessary and sufficient condition for semi-stability of an internal equilibrium is that there be a single positive eigenvalue and at least one negative eigenvalue of $W$ Let us put $\Delta_{p}, p=1, \ldots, k$ for the determinant of the $p \times p$ north-west corner truncated matrix of $W$. Kingman notes that, if none of these are zero, the above necessary and sufficient condition can be re-expressed by requiring

$$
(-1)^{p} \Delta_{p}<0, \quad p=1, \ldots, k .
$$

Without the initial proviso that none of the $\Delta_{p} \neq 0,(8)$ is therefore only a sufficient condition for semi-stability. Indeed, not only does it imply non-singularity of $W$, but a great deal more.

Since for a non-singular $W$, as we have seen, an internal equilibrium point is semi-stable if and only if it is stable, for a non-singular $W$, we have as necessary and sufficient condition for internal stability: that there be a single positive eigenvalue of $W$ (Kingman).

It is important to make a distinction at this stage between (8) and a necessary and sufficient condition for negative definiteness of the quadratic form $\boldsymbol{x}^{T} W \boldsymbol{x}$. Indeed,

$$
x^{T} W x<0, \quad x \neq 0
$$

is equivalent to (Gantmacher and Krein, 1960)

$$
(-1)^{p} \Delta_{p}>0, \quad p=1, \ldots, k \text {. }
$$

Indeed, the distinction between (8) and (10) is emphasised by the fact that in a negative-definite quadratic form (9), $W$ must have all its eigenvalues strictly negative, and we know that $W$ has a positive eigenvalue, since it is not only symmetric but non-negative in our setting. This distinction between (8) and (10) is evidently caused by the innocent requirement that in (6), we suppose also that $\boldsymbol{\delta}^{T} \mathbf{1}=0$, and under this constraint the situation is consistent, while the unconstrained quadratic form (9) cannot be negative definite.

The efforts at expressing stability of an internal equilibrium point by means of a determinantal criterion such as (8) for a non-singular matrix $W$ have a slightly vexed history. Generally speaking, such efforts to "build in " the condition $\boldsymbol{\delta}^{T} \mathbf{1}=0$ in the consideration of positive definiteness of the matrix $W$, have led to determinantal criteria similar to (8) and (10), but in terms of a modified matrix (not $W$ itself), say $V$, for which (6) is equivalent to the unconstrained requirement:

$$
\boldsymbol{x}^{T} V x<0 \text { for all } \boldsymbol{x} \neq \mathbf{0}
$$


so that $(10)$, with the $\Delta_{p}$ referring to $V$, is equivalent. Three such sets of criteria for non-singular $W$ are reviewed and shown to be equivalent to each other by Mandel (1970). Aesthetically, the criterion (8), which we may attribute to Kingman (1961 $b$ ), seems most pleasing.

We conclude our discussion of internal existence and stability with several sentences from Ewens (1969, p. 11), which reveal the extent of confusion existing even in the mathematically inclined population genetic literature:

"... there can be at most one equilibrium point at which $p_{i}>0$. This equilibrium point, if it exists, will be stable if the matrix $W=\left\{w_{i j}\right\}$ has exactly one positive eigenvalue and at least one negative eigenvalue. ..."

\section{Boundary equilibrium POINTS}

A boundary equilibrium point $\boldsymbol{p}$ is an equilibrium point containing at least one zero entry. In considering vectors $\boldsymbol{p}$ with prescribed entries zero (in an investigation of such peripheral equilibrium points), it is evident from (1) that this is tantamount to ignoring the corresponding alleles, and working with an appropriately diminished fitness matrix, for which the existence-stability problem now becomes one for internal equilibria. It is also clear that if we consider the case where we permit at most two entries of the $k$-length vector $\boldsymbol{p}$ be non-zero, we are in the well-known and extensively studied di-allelic case, which has an internal stable equilibrium point (balanced polymorphism), in case of heterozygote advantage, and otherwise stable boundary points of homozygosity.

Should it happen that the determinant of each principal minor of $W$ is non-zero, it is readily shown (Tallis, 1966) there is at most a totality of $2^{k}-1$ equilibrium points for the $k$-allele system, including boundary points.

The number comes about from the fact that for each possible distinct subset of alleles to be deleted, there is for the resulting system at most one equilibrium point.

Tallis' paper is concerned to an extent with the problem of enumeration or determination of equilibrium points-he at least alludes to the use of a generalised inverse.

\section{Mathematical appendix}

We present here the aspects, in appropriately simplified form, of the theory of generalised inverse which are needed in the preceding discussion.

I. Consider a $k \times k$ matrix of rank $r<k$. By moving $r$ linearly independent columns of it to the first $r$ positions, and then adjusting the order of rows, it may be put into the form

$$
A=\left[\begin{array}{ll}
B & C \\
D & E
\end{array}\right],
$$

where $B$ is $r \times r$ and non-singular. Now there exists, by linear dependence of each of the last $k-r$ columns on the first $r$, an $r \times(k-r)$ matrix $\Delta$ such that

$$
\left[\begin{array}{l}
B \\
D
\end{array}\right] \Delta=\left[\begin{array}{l}
C \\
E
\end{array}\right],
$$

whence $\Delta=B^{-1} C$ and hence $E=D B^{-1} C$ in $A$. 
Suppose now that

$$
\boldsymbol{b}=\left[\begin{array}{l}
b_{1} \\
b_{2}
\end{array}\right], \quad \text { where } b_{1} \text { is } r \times 1, b_{2} \text { is }(k-r) \times 1
$$

is a vector of known constants, and the system of linear equations $A \boldsymbol{x}=\boldsymbol{b}$ has a solution $\boldsymbol{x}$; partitioning $\boldsymbol{x}$ appropriately

i.e.

$$
\left[\begin{array}{ll}
B & C \\
D & E
\end{array}\right]\left[\begin{array}{l}
x_{1} \\
x_{2}
\end{array}\right]=\left[\begin{array}{l}
b_{1} \\
b_{2}
\end{array}\right]
$$

$$
\begin{aligned}
& b_{1}=B x_{1}+C x_{2} \\
& b_{2}=D x_{1}+E x_{2}=D x_{1}+D B^{-1} C x_{2}=D B^{-1}\left(B x_{1}+C x_{2}\right)=D B^{-1} b_{1}
\end{aligned}
$$

so that

$$
\boldsymbol{b}_{2}=D B^{-1} \boldsymbol{b}_{1} \text {. }
$$

A direct check now reveals that one solution to $A \boldsymbol{x}=\boldsymbol{b}$ is

$$
\boldsymbol{x}=\left[\begin{array}{c}
B^{-1} b_{1} \\
0
\end{array}\right]=\left[\begin{array}{ll}
B^{-1} & 0 \\
0 & 0
\end{array}\right] \boldsymbol{b} .
$$

Indeed, the $k \times k$ matrix of the extreme right-hand side is a generalised inverse $A^{-}$, in the sense of Rao (1965, Section $\left.1 \mathrm{~b}, 5\right)$, and the above discussion is implicit in his much contracted notes. We note also (but without proof) the property mentioned in this reference, that if $H=A^{-} A$, the general solution of $A \boldsymbol{x}=\boldsymbol{b}$ is given by

$$
A^{-b}-(H-I) z,
$$

where $z$ is an arbitrary vector (so we are free to put $-z$ for $z$, as Rao does). Using our specific form of generalised inverse and writing $z=\left(z_{1}^{\prime}, z_{2}^{\prime}\right)^{\prime}$ this becomes

$$
\left[\begin{array}{c}
B^{-1} b_{1} \\
0
\end{array}\right]-\left[\begin{array}{c}
B^{-1} C z_{2} \\
-z_{2}
\end{array}\right]=\left[\begin{array}{c}
B^{-1}\left(b_{1}-C z_{2}\right) \\
z_{2}
\end{array}\right],
$$

where $z_{2}$ is $(k-r) \times 1$ and arbitrary.

II. If we assume additionally at the outset that the matrix with which we deal is symmetric, let us begin by a shift of linearly independent columns to the first $r$ positions as before, but then carry out a corresponding rearrangement of rows. Then we know $A$, and so $B$, in (A.1) is symmetric, but it is no longer certain that $B$ is non-singular. However, we notice that, by the symmetry of $A$, the last $k-r$ rows of $A$ may be expressed linearly in terms of the first $r$, and hence the rows of $D$ may each be expressed linearly in terms of the rows of $B$. Hence the rows of $B$ are linearly independent, for if not the rank of the matrix $\left[\begin{array}{l}B \\ D\end{array}\right]$ would be less than $r$. Thus $B$ is nonsingular, and the rest follows as before. 
III. (To Section 2.) Suppose both $z_{2}>0$ and $z_{2}^{*}>0$ define internal equilibrium points $p>0$ and $p^{*}>0$ by means of (4): we have at each of these points

$$
\mu(p)=1 / g\left(z_{2}\right), \quad \mu\left(p^{*}\right)=1 / g\left(z_{2}^{*}\right),
$$

where the generic expression for $g$ is given by

$$
g\left(z_{2}\right)=1^{T} A_{11}^{-1}\left(1-A_{12} z_{2}\right)+1^{T} z_{2},
$$

where not all vectors 1 are necessarily of same length. Partitioning the equilibrium-point vectors according to I above, and using (2) in partitioned form of Section 2, we deduce from (A.2), where $b=1$, that

$$
1=A_{21} A_{11}^{-1} 1
$$

so from (A.3) and using the symmetry of $W$

$$
g\left(\mathrm{z}_{2}\right)=\mathbf{1}^{T} A_{11}^{-1} \mathbf{1}=g\left(\mathrm{z}_{2}^{*}\right) .
$$

\section{REFERENCES}

ATKINSON, F. V., WATTERSON, G. A., AND MORAN, P. A. P. 1960. A matrix inequality. Quarterly Fournal of Mathematics (Oxford, 2nd ser.), 11, 137-140.

CORMACK, R. M. 1964. A boundary problem arising in population genetics. Biometrics, 20, 785-793.

CRow, J. F., AND KIMURA, M. 1970. Introduction to Population Genetics. Harper and Row, New York.

EWENs, w. J. 1969. Population Genetics. Methuen, London.

FELLER, w. 1960. A geometrical analysis of fitness in triply allelic systems. Mathematical Biosciences, 5, 19-38.

GANTMACHER, F. R., AND KREIN, M. G. 1960. Oszillationmatrizen, Oszillationskerne und Kleine Schwingungen Mechanischer Systeme. Akademie Verlag, Berlin. (Originally as: Ostsillyatsionnye Matritsy $i$ Yadra i Malye Kolebaniya Mechanicheskikh Sistem. G.I.T.-T.L., 2nd edn., Moscow-Leningrad, 1950; also in a rare English translation: Oscillation Matrices and Kernels and Small Vibrations of Mechanical Systems. Translation Series: AEC-tr-4481, Physics. United States Atomic Energy Commission. Office of Technical Information, 1961.)

KIMURA, м. 1956. Rules for testing stability of a selective polymorphism. Proceedings of the National Academy of Sciences U.S.A., 42, 336-340.

KINGMAN, J. F. C. 1961a. On an inequality in partial averages. Quarterly fournal of Mathematics (Oxford, 2nd ser.), 12, 78-80.

kingman, J. F. c. 1961b. A mathematical problem in population genetics. Proceedings of the Cambridge Philosophical Society, 57, 574-582.

LEVENE, H., PAVLOVSKY, O., AND DOBZHANSKY, TH. 1954. Interaction of the adaptive values in polymorphic experimental populations of Drosophila pseudoobscura. Evolution, $8,335-349$.

LI, c. c. 1955. The stability of an equilibrium and the average fitness of a population. American Naturalist, 89, 281-296.

II, c. c. 1967. Genetic equilibrium under selection. Biometrics, 23, 397-484.

MANDEL, S. P. H., AND HUGHES, I. M. 1958. Change in mean viability at a multiallelic locus in a population under random mating. Nature, 182, 63-64.

MANDEL, s. P. H. 1959. The stability of a multiple allelic system. Heredity, 13, 289-302.

MANDEL, S. P. H. 1970. The equivalence of different sets of stability conditions for multiple allelic systems. Biometrics, 26, 840-845.

MULHollaND, H. P., AND SMTTH, C. A. B. 1959. An inequality arising in genetical theory. American Mathematical Monthly, 66, 673-683.

oWEN, A. R. G. 1953. A genetical system admitting of two distinct stable equilibria under selection. Heredity, 7, 97-102. 
OWEN, A. R. G. 1954. Balanced polymorphism of a multiple allelic series. Caryologia, 6 (Supplement), 1240-1241. (Atti del IX Congr. Internazionale di Genetica.)

oweN, A. R. G. 1959. Mathematical models for selection, in Natural Selection in Human

Populations (Vol. II, Symp. Soc. Study of Human Biol.; D. F. Roberts and G. A.

Harrison, eds.). Pergamon, New York, pp. 11-16.

PENROSE, L. s., SMITH, s. M., AND SPROTT, D. A. 1956. On the stability of allelic systems, with special reference to haemoglobins A, S, and C. Annals of Human Genetics, 21, 90-93.

RAO, G. R. 1965. Linear Statistical Inference and its Applications. Wiley, New York.

SGHEUER, P. A. G., AND MANDEL, s. P. H. 1959. An inequality in population genetics. Heredity, $13,519-524$.

SENETA, E. 1973. Non-Negative Matrices. Allen and Unwin, London.

TALLIs, G. M. 1966. Equilibria under selection for $k$ alleles. Biometrics, 22, 121-127.

WEIR, B. s. 1970. Equilibria under inbreeding and selection. Genetics, 65, 371-378. 